\title{
Comparison of Cognitive Performances During a Placebo Period and an Atypical Antipsychotic Treatment Period in Schizophrenia: Critical Examination of Confounds
}

\author{
Thomas W Weickert*,', Terry E Goldberg', Stefano Marenco', Llewellyn B Bigelow', Michael F Egan' and \\ Daniel R Weinberger'
}

'Clinical Brain Disorders Branch, National Institute of Mental Health, National Institutes of Health, Bethesda, MD, USA

\begin{abstract}
Although previous studies report cognitive improvement following atypical antipsychotic administration in schizophrenia (SC), few placebo-controlled within-subject studies with examination of confounds (symptom reduction, cooperation, learning, and outliers) have been reported. The present study examines the effects of atypicals and confounds upon cognition in SC. The hypothesis tested was that relative to placebo, atypicals as a general class of medication would elicit cognitive improvement in SC. In all, 19 patients with SC (I5 males) completed the double-blind, counterbalanced, randomized within-subject study of the effects of atypical antipsychotics (risperidone, clozapine, olanzapine, or quetiapine) vs placebo administration upon cognitive performance in the domains of executive function, attention, memory, language, visual perception, and general intellect. Significant cognitive improvement during atypical antipsychotic administration relative to placebo withdrawal occurred in most cognitive domains with robust improvements in intelligence $(p=0.00 \mathrm{I})$, memory $(p=0.0009)$, and fluency $(p<0.002)$ even after outliers and unmotivated performances were excluded. These findings suggest that relative to placebo withdrawal, atypicals improve cognitive performance in SC. However, this finding may not be specific to atypicals, since analogous studies of typicals have not been performed.

Neuropsychopharmacology (2003) 28, |49|-1500, advance online publication, I I June 2003; doi:10.1038/sj.npp. I3002 I6
\end{abstract}

Keywords: schizophrenia; placebo; cognition; antipsychotic agents; learning; dopamine

\section{INTRODUCTION}

Schizophrenia (SC) is a psychiatric disorder characterized by a range of cognitive deficits in the domains of executive function, working memory, attention, and memory (Palmer et al, 1997; Weickert et al, 2000). Alteration in the function of the dopamine neurotransmitter system has been hypothesized, at least in part, to underlie the expression of psychotic symptoms such as delusions, hallucinations, disordered thought, and avolition (Seeman, 1987). Evidence supporting the dopamine hypothesis of SC has been based indirectly on the finding that administration of potent dopamine D2 receptor blocking agents have been successful in the attenuation of psychotic symptoms (Cole et al, 1964). Although these so-called 'typical' neuroleptic agents have been relatively successful in the reduction of psychotic

*Correspondence: Dr TW Weickert, Clinical Brain Disorders Branch, NIMH/NIH, Building 10 Room 4N 202, MSC 1379, Bethesda, MD 20892, USA, Tel: + I 30 I 435 7645, Fax: + I 30 I 4807795 ,

E-mail: weickert@intra.nimh.nih.gov

Received 07 November 2002; revised 26 March 2003; accepted 03 April 2003

Online publication: 22 April 2003 at http://www.acnp.org/citations/ Npp02-0403R/default.pdf symptoms, they frequently cause adverse side effects, such as tardive dyskinesia, and have been thought not to improve performance on tests designed to assess the cognitive domains of executive function, attention, and memory (for a review see Goldberg and Weinberger, 1996).

More recently, a new generation of 'atypical' antipsychotic medication has been developed that displays lower affinity for dopamine D2 receptors and greater affinity for other receptor sites such as serotonin and acetylcholine (Farde et al, 1989; Meltzer, 1989; Meltzer et al, 1989a, b, c; Richelson, 1984). In general, the atypical antipsychotic medications produce an equivalent reduction in symptoms compared to typical neuroleptic medications and generally cause fewer undesirable side effects (see Geddes et al, 2000 for a review; however, see also Davis and Chen, 2002, 2001 for an alternate view suggesting superior symptom reduction for atypicals).

In their review of 15 studies examining the effects of atypical antipsychotic medication on the cognitive abilities of patients with SC, Keefe et al (1999), after correcting for multiple comparisons, determined that nine of the 15 studies found significant cognitive improvement for at least one test measure. Several meta-analytic procedures applied to the 15 studies revealed that atypical antipsychotics were 
more effective than typical neuroleptics in producing cognitive improvement (Keefe et al, 1999). However, enthusiasm for these results must be tempered since rigorous attempts to rule out or control for possible confounds (such as learning associated with repeated testing, lack of cooperation and motivation, symptom reduction, and inclusion of outliers) that may have influenced the results were not conducted in the majority of these studies.

Additionally, the majority of previous studies of atypical antipsychotics were between group comparisons, allowing for the possibility that cohort or baseline differences might have influenced the results. Few studies examined the effect of atypical antipsychotics in comparison with a medicationfree period using a within-subject design (Hagger et al, 1993; Potkin et al, 2001; Serper and Chou, 1997; Zahn et al, 1994). With one exception, (Potkin et al, 2001), patients were always tested in the medication-free phase initially, thereby providing a potential practice or learning advantage for the medication phase. Additionally, with the exception of Potkin et al (2001), previous studies did not use a particularly long medication-free phase (greater than 7 days) to effectively reduce dopamine blockade and restore dopamine activity to that generally associated with the occurrence of florid symptoms.

While the atypical antipsychotics appear to produce differential improvement of cognitive abilities in patients with SC (Bilder et al, 2002; Buchanan et al, 1994; Fujii et al, 1997; Gallhofer et al, 1996; Hagger et al, 1993; Hoff et al, 1996; Lee et al, 1994; Meltzer, 1992; Meltzer and McGurk, 1999; Potkin et al, 2001; Purdon et al, 2001), the aim of the current study was not to describe unique profiles of cognitive enhancement associated with specific atypicals as has been shown previously, but rather to address the heretofore unanswered question of whether certain confounding variables contribute to the cognitive improvement associated with the administration of atypicals. Therefore, the objectives of the current study were two-fold: (1) to examine the effects of a general class of antipsychotic medication (atypicals) upon general cognitive abilities encompassing the domains of executive function, attention, memory, language, and perception in a well-diagnosed, partially treatment-responsive, yet chronically impaired, cohort of patients with SC by comparing performance during atypical administration to performance during a relatively extended medication-free period using a within-subject, double-blind, placebo-controlled, counterbalanced design (allowing for control of learning associated with repeated testing, which may serve as a confounding variable), and (2) to perform a critical examination of potentially confounding factors (such as learning, lack of motivation, outliers, and symptom reduction) that may bias results in favor of antipsychotic enhancement of cognition. Our working hypothesis was that there would be cognitive improvement in patients with SC administered atypical antipsychotics relative to the same patients when they were withdrawn from all antipsychotics and placed on placebo. Based on the Keefe et al (1999) meta-analysis, measures of verbal fluency, executive function, and attention would be most likely to display improvements following administration of atypical antipsychotic medications.

\section{METHOD}

\section{Patients}

A diagnosis of SC or schizoaffective disorder was independently made by at least two board certified psychiatrists blind to the neuropsychological performance through application of DSM IV criteria during chart review and interview. Patients receiving concurrent axis I psychiatric diagnoses or having a history of recent substance abuse, head injuries with concomitant loss of consciousness, seizures, central nervous system infection, diabetes, or hypertension were excluded from the study. A total of 31 inpatients recently recruited through national advertisement for purposes of the current research study met the eligibility criteria. Eight patients withdrew consent and four were withdrawn due to marked symptom exacerbation during placebo administration and 19 (15 males and four females) completed the study at an in-patient psychiatry unit of either the Warren G Magnuson Clinical Center at the National Institute of Mental Health (NIMH), Bethesda, MD, or the NIMH Neuroscience Center at St Elizabeths Hospital, Washington, DC. Nine patients were classified with paranoid subtype of SC, eight with undifferentiated subtype, one with residual subtype, and one as schizoaffective depressed. The patients had a mean age of 33.8 years (standard deviation, $S D=6.9$ ), a mean number of years of education equal to $13.5(\mathrm{SD}=1.6)$, a mean age of onset of 22.3 years $(\mathrm{SD}=4.2)$, a mean duration of illness of 11.5 years $(\mathrm{SD}=8.8)$, and a mean Wide Range achievement TestRevised (Jastak and Wilkinson, 1984) Reading standard score (a measure of preserved pronunciation skills frequently used as an estimate of premorbid intellect) of 105.6 $(\mathrm{SD}=11.9)$. All patients provided informed written consent before participating in this study. This study was reviewed and approved by the Institutional Review Board of the National Institute of Mental Health.

\section{Design}

Using a double-blind within-subject design the study was divided into two phases. During one phase, patients were administered atypical antipsychotics: four received risperidone, three received clozapine, two received clozapine and risperidone, one received quetiapine fumarate, one received quetiapine fumarate and olanzapine, seven received olanzapine, and one received olanzapine and risperidone. See Table 1 for the daily dosages of atypical antipsychotic and adjunctive medications administered during the active medication phase. Antipsychotic medication was chosen based on availability of coded inactive forms and after consultation between clinician and patient. During the other phase, patients were administered placebo (or placebos in the case of administration of multiple antipsychotics and adjunctive medications during the active medication phase) and no adjunctive medications. To correct for potential learning effects, each patient was randomly assigned to one of two orders: half of the patients were first assessed on cognitive measures while receiving antipsychotics followed by cognitive assessment during the administration of placebo (the 'on-off' group); the other half of the patients underwent cognitive assessment while receiving placebo 
Table I Antipsychotics, Adjunctives, and Daily Dosages (in mg) Administered During the 'Active' Medication Phase

\begin{tabular}{|c|c|c|c|c|}
\hline Patient & Antipsychotic I & Antipsychotic 2 & Adjunctive I & Adjunctive 2 \\
\hline Ol & Risperidone 5 & - & - & - \\
\hline 02 & Clozapine 200 & Risperidone 4 & - & - \\
\hline 03 & Quetiapine 400 & - & - & - \\
\hline 04 & Olanzapine 10 & - & - & - \\
\hline 05 & Clozapine 250 & - & Valproic acid 500 & - \\
\hline 06 & Risperidone 6 & - & Valproic acid 1000 & Benztropine 2 \\
\hline 07 & Quetiapine 700 & Olanzapine 20 & - & - \\
\hline 08 & Clozapine 350 & Risperidone 6 & - & - \\
\hline 09 & Olanzapine 20 & - & Valproic acid 1500 & - \\
\hline 10 & Olanzapine 25 & - & - & - \\
\hline | | & Olanzapine 20 & - & - & - \\
\hline 12 & Olanzapine 10 & - & - & - \\
\hline 13 & Olanzapine 20 & - & Valproic acid 750 & - \\
\hline 14 & Olanzapine 5 & - & - & - \\
\hline 15 & Olanzapine 15 & Risperidone 4 & - & - \\
\hline 16 & Risperidone 6 & - & - & - \\
\hline 17 & Clozapine 300 & - & - & - \\
\hline 18 & Risperidone 8 & - & - & - \\
\hline 19 & Clozapine 350 & - & Fluoxetine 60 & - \\
\hline
\end{tabular}

first followed by cognitive assessment during administration of antipsychotics (the 'off-on' group). During the period of administration of inactive medication, patients received placebo and were 'medication free' for a period of between 3 and 4 weeks (whole sample mean $=24.5$ days, $\mathrm{SD}=5.0$; 'on-off group mean $=24.5$ days, $\mathrm{SD}=6.1$; 'offon' group mean $=24.4$ days, $\mathrm{SD}=3.9$ ) before cognitive assessment was obtained in order to allow sufficient time for antipsychotics to clear their system. The mean number of days between cognitive assessments in the two conditions (antipsychotics vs placebo) was 84.2 days $(\mathrm{SD}=39.9)$. Assessments were made blind to the medication status of the patients. During the administration of atypical antipsychotic medication patients were 'stabilized' on an atypical antipsychotic medication for a period of between 6 and 7 weeks (whole sample mean $=47.5$ days, $S D=17.5$; 'on-off group mean $=43.3$ days, $\mathrm{SD}=15.5$; 'off-on' group mean $=52.2$ days, $S D=19.3$ ) before cognitive assessments were performed.

\section{Cognitive Tests}

A comprehensive battery of tests encompassing the cognitive domains of executive function, attention, memory, perception, and general intellect, previously demonstrated to be adversely effected in patients with SC (Palmer et al, 1997; Weickert et al, 2000), was administered twice by a trained psychologist (TWW). Scoring followed standardized procedures. The Wisconsin Card Sorting Test (WCST) (Heaton et al, 1993) was administered as a measure of executive function. The $N$-back ( $0-3$ back) test (Goldberg et al, in press; Callicott et al, 1999) was administered as a measure of working memory. The Distractibility version of the Gordon Continuous Performance Test (CPT) (Gordon, 1987; Gordon et al, 1996) was administered as a test of attention. Logical Memory I and II, Verbal Paired Associates I and II, Visual Reproduction I and II, and Digits Forward from the Wechsler Memory Scale-Revised (WMS-R) (Wechsler, 1987), and List A trials 1-5, Free
Recall after a Long Delay, and Delayed Recognition from the California Verbal Learning Test (CVLT) (Delis et al, 1987) were administered as tests of episodic memory. Benton Line Orientation (BLO) and Benton Facial Recognition (BFR) (Benton et al, 1983) were administered as measures of visual processing ability. Letter and Category fluency tests (Spreen and Strauss, 1991) were administered as tests of language skills and lexical access. Forms A and B of the Trail Making Test (Reitan, 1986) were administered as tests of oculomotor tracking and psychomotor speed. A four-subtest version of the Wechsler Adult Intelligence Scale-Revised (WAIS-R) (Kaufman, 1990; Missar et al, 1994; Wechsler, 1981) consisting of the Arithmetic, Similarities, Picture Completion, and Digit Symbol Substitution tests was administered to obtain an estimate of current Full-Scale Intelligence Quotient (FSIQ).

\section{Symptom Assessment}

Symptoms were assessed weekly during the antipsychotic administration phase and the antipsychotic-free placebo phase by members of the nursing staff who were trained in the administration and scoring of the Positive and Negative Symptoms Scale (PANSS) (Kay et al, 1987). The assessment closest to the cognitive testing dates in each phase of the protocol was used to obtain indices of positive symptoms, disorganization, negative symptoms, general, and total scale scores.

\section{Statistical Analyses}

The first analytic procedure (MANOVA) included all data collected from 16 out of 19 patients in order to maximize the $N$ since three patients had missing data for some variables. Raw scores were first converted to $z$-scores to enable comparisons among different assessment measures. A three-way (medication status $\times$ order $\times$ test) repeated measures MANOVA was applied to the data set in which drug status (on $v s$ off) and test were within-subject variables 
and order (on antipsychotic then off $v s$ off antipsychotic then on) was considered a between-subject class variable. We considered a significant drug status $\times$ test interaction to be the critical comparison that would permit the use of post hoc matched pairs $t$-tests to assess relative differences in cognitive change and symptom change during the administration of atypical antipsychotics and placebo. Determination of the effect size for each of the cognitive measures was used to assess the magnitude of the effect of atypical antipsychotic administration $v s$ placebo. Additionally, matched paired $t$-tests of cognitive assessments during Time $1 v s$ Time 2 regardless of medication status were performed to determine whether learning or practice effects were present.

Further methods to determine the robustness of the effect entailed: (1) application of a Bonferroni correction procedure (each of the $t$-tests results were considered to be significant after consistently and uniformly applying a Bonferroni correction for multiple comparisons using $p<0.002$ unless otherwise noted), (2) exclusion of outliers by applying a $-1.5 \mathrm{SD}$ cutoff criteria to the entire data set, (3) exclusion of clinically determined poorly motivated or otherwise invalid performances, (4) correlation between symptom and cognitive changes, and (5) determination of the percent of patients' performances that were normalized following administration of atypical antipsychotic medication. Exclusion of outliers involved routinely applying a cutoff score of $-1.5 \mathrm{SD}$ units to the entire data set (including both antipsychotic and placebo conditions) for all patients blind to medication status. An individual score on any test that was 1.5 SD units below the sample mean was omitted from the analysis. In this sense any poorly performing outliers that may have biased the results in favor of finding a difference due to antipsychotics were excluded. Exclusion of clinically determined invalid performances involved a detailed review of the entire data set (including both antipsychotic and placebo conditions) for each patient by TWW and TEG, blind to medication status, to determine whether each patient exerted a 'reasonable' amount of effort during each test. Such effort was assessed on the clinical basis of (1) whether or not the patient was providing responses rather than simply providing statements such as 'I don't know' and obtaining scores of ' 0 ', (2) whether the patients' responses appeared to be intentionally directed to contradict the instructions of the experimenter (this occurred only in one instance), and (3) whether each patient's responses appeared to be inconsistent with their own performance or typical performances exhibited by most patients. If a patient was determined not to be exerting a reasonable amount of mental effort during any given test then the data collected during that session for that specific test was omitted from the analysis. For either exclusionary method described above, when data were removed for a patient on a specific parameter under a given condition (on or off medication) data from the corresponding condition was also removed from the analysis in order to allow for a matched comparison. Correlation and regression analyses were performed between difference scores for the cognitive testing sessions and change scores for symptoms to determine whether changes in symptoms or IQ may be related to changes in cognitive performance. In order to maximize the number of patients in our correlations we used pairwise deletion of missing data resulting in variable numbers of patients ranging from 12 to 17 on which correlations were based.

The percentage of patients whose cognitive performances were 'normalized' by the administration of atypical antipsychotic medications was calculated using two methods. The first stringent method determined the number of patients whose impaired scores (during placebo) on test measures improved by at least one standard deviation unit and fell within the average range as specified by published norms for those tests. For example, using this stringent method, a patient's estimated FSIQ score on WAIS-R would have to increase by at least 15 points from placebo to medication phase and be above 85 in order to be considered normalized. A second lenient method was also employed. Using the lenient method, each patient's scores during the placebo withdrawal period and during the atypical antipsychotic medication period were inspected to determine whether the scores improved to within one standard deviation of the mean of normal healthy subjects as described in previously published norms for each test measure. For example, using this lenient method a patient's estimated FSIQ score on WAIS-R would have to increase from below 85 to above 85 in order to be considered normalized.

\section{RESULTS}

\section{Cognition and Confounds}

Patients with SC uniformly and consistently demonstrated improvement in performance across all cognitive domains when administered atypical antipsychotics relative to their performance when they received placebo during a withdrawal period. Results of a three-way repeated measures MANOVA on the whole sample of patients demonstrated no significant effect of order (off then on $v s$ on then off), $\mathrm{F}(1,14)=0.01, p=0.92$, a significant main effect of drug status (on $v s$ off), $\mathrm{F}(1,14)=48.81, p<0.04$, no interaction between order and test, $\mathrm{F}(16,224)=7.40, p=1.00$, and a significant interaction between drug status and test, $\mathrm{F}(16,224)=61.41, p<0.001$. The latter results suggest that being on or off atypical antipsychotic medication had a differential effect upon the patients' performance on tests of different cognitive domains (ie not all tests improved to the same degree). Processes involving RT (CPT and N-back) or updating and set shifting ( $N$-back, WCST perseverative errors) generally improved less, while memory and language production tasks improved more, as can be seen from Table 2 .

Results of individual matched pairs $t$-tests of patients' performance on and off medication demonstrated that drug administration significantly improved performance on measures of general intellect (WAIS-R FSIQ), memory (General Memory Index from the WMS-R), and word production (Letter and Category fluency) even after the Bonferroni correction (see Table 2). Although other measures did not reach statistical significance after correction for multiple comparisons, nearly all these other measures improved, albeit to varying degrees, with the administration of atypical antipsychotic medication. In general, effect sizes were moderate to large (see Table 3 ). 
Table 2 Mean Cognitive Test Scores, PANSS scores, and SDs for 19 Patients with Schizophrenia During Administration of Atypical Antipsychotic Medication and During a Medication-Free Period

\begin{tabular}{|c|c|c|c|c|}
\hline & $\begin{array}{l}\text { Administered } \\
\text { antipsychotics }\end{array}$ & $\begin{array}{l}\text { Medication } \\
\text { free }\end{array}$ & $T$ & $p$ \\
\hline \multicolumn{5}{|l|}{ PANSS } \\
\hline Positive symptoms & | 3.0 (3.9) & |7.4 (5.1) & 3.14 & 0.006 \\
\hline Negative symptoms & $15.9(6.8)$ & $20.1(6.8)$ & 2.36 & 0.03 \\
\hline \multicolumn{5}{|l|}{ WAIS-R } \\
\hline FSIQ & $96.21(11.76)$ & $84.26(16.03)$ & 3.81 & $0.00 \mid *$ \\
\hline \multicolumn{5}{|l|}{ CPT distractibility } \\
\hline Correct & $24.65(8.30)$ & $20.24(10.45)$ & 1.64 & 0.12 \\
\hline Commission errors & $3.12(4.83)$ & $7.00(\mid 4.84)$ & 1.05 & 0.31 \\
\hline Reaction time & $463.53(143.04)$ & $503.53($ ( 34.58$)$ & 1.35 & 0.19 \\
\hline \multicolumn{5}{|l|}{ Trail Making Test } \\
\hline Form A & $39.59(14.47)$ & $51.94(32.25)$ & 2.11 & 0.05 \\
\hline Form B & 73.08 (15.78) & | | 3.92 (85.32) & 1.82 & 0.09 \\
\hline \multicolumn{5}{|l|}{ WMS-R } \\
\hline Digit span forward & $7.7(1.7)$ & $7.2(2.7)$ & 0.89 & 0.39 \\
\hline General Memory Index & $95.75(19.85)$ & $82.88(26.26)$ & 4.11 & $0.0009 *$ \\
\hline Delayed Recall Index & $94.00(20.52)$ & $84.59(24.06)$ & 2.80 & 0.01 \\
\hline \multicolumn{5}{|l|}{ CVLT } \\
\hline List $A$ trials $\mid-5$ & $47.39(12.29)$ & $37.06(20.22)$ & 3.41 & 0.003 \\
\hline Long Delay Free Recall & $10.1 \mid(3.92)$ & $8.28(5.26)$ & 2.15 & 0.05 \\
\hline Slope & $1.21(0.67)$ & $0.83(0.63)$ & 2.13 & 0.05 \\
\hline Recognition-Discriminability & $90.83(10.97)$ & $84.17(14.50)$ & 2.60 & 0.02 \\
\hline BLO & $24.94(4.54)$ & $21.17(8.40)$ & 2.28 & 0.04 \\
\hline BFR & $47.00(4.05)$ & $45.44(5.62)$ & 1.29 & 0.22 \\
\hline \multicolumn{5}{|l|}{ WCST } \\
\hline Total number of categories & $3.94(3.5 \mathrm{I})$ & $2.89(3.18)$ & 2.33 & 0.03 \\
\hline Percent perseverative errors & $23.53(16.50)$ & $27.04(19.41)$ & 0.72 & 0.48 \\
\hline Letter fluency & $36.56(13.29)$ & $28.11(15.62)$ & 3.59 & $0.002 *$ \\
\hline Category fluency & $40.17(12.86)$ & $30.17(15.24)$ & 5.64 & $0.00003 *$ \\
\hline \multicolumn{5}{|l|}{ Working memory (N-back) } \\
\hline \multicolumn{5}{|l|}{ One back } \\
\hline Number correct & $58.93(27.62)$ & $49.00(26.83)$ & 1.43 & 0.18 \\
\hline Reaction time & $808.45(309.17)$ & $743.72(310.11)$ & 1.00 & 0.34 \\
\hline \multicolumn{5}{|l|}{ Two back } \\
\hline Number correct & $47.14(24.38)$ & $42.93(19.79)$ & 1.04 & 0.32 \\
\hline Reaction time & $805.38(327.69)$ & $809.73(300.46)$ & 0.06 & 0.96 \\
\hline \multicolumn{5}{|l|}{ Three back } \\
\hline Number correct & 41.57 ( 18.29$)$ & $36.29(15.17)$ & 2.03 & 0.06 \\
\hline Reaction time & $801.58(265.5 \mid)$ & $780.52(294.86)$ & 0.41 & 0.69 \\
\hline
\end{tabular}

Notes: SDs are in parentheses. The number of patients assessed for any given measure ranged between 13 and 19 due to patient compliance and ability. The FSIQ of the WAIS-R has a mean score of I00 and a SD of I5. For the distractibility version of the CPT, mean number of correct responses and commission errors are provided and reaction time is given in milliseconds. For the Trail Making Test Forms A and B, values refer to time elapsed (in seconds) to complete task. For the WMS-R, index scores have a mean score of 100 and an SD of I5, for Digit Span Forward the values refer to the number of trials correctly repeated. For the CVLT, the mean number of correct responses is given for all measures with the exception of Slope and Recognition-Discriminability, for which the increment in words recalled per trial and the percent correct are given, respectively. For the BLO and BFR tasks, mean number of correct responses are provided. For the WCST, mean values are provided for all measures. On the Letter and Category fluency tasks, mean number of words produced are provided. On the working memory ( $N$ back task), mean number correct and reaction time in milliseconds are provided. For the PANSS scores mean values are provided for positive and negative symptoms. multiple comparisons at $p<0.05$.

The within-subject comparison of Time 1 vs Time 2 assessments without regard to medication status indicated a lack of learning effects. Only five of the 34 Time $1 v s$ Time 2 comparisons met traditional significance levels $(p<0.05)$; significant differences were not consistent within a given cognitive domain and none withstood Bonferroni correction.

The results of the analyses following exclusion of outliers and the removal of invalid data considered to be compatible with a lack of effort on the part of individual patients during specific tests were virtually identical to the results presented in Table 2 (results available upon request). Application of these procedures resulted in the exclusion of data from 0 to 3 patients for any given parameter. Regarding the percent of patients whose cognitive performance was normalized following administration of atypical antipsychotics, using the stringent method of determining normalization, on average, $20.4 \%$ of the patients' scores were normalized over 
Table 3 Effect Sizes Determined by Comparison of Cognitive Performances of 19 Patients with Schizophrenia Following Administration of Atypical Antipsychotics and Placebo after Applying Different Exclusionary Criteria

\begin{tabular}{lccc}
\hline Assessment measure & $\begin{array}{c}\text { Whole } \\
\text { sample }\end{array}$ & $\begin{array}{c}\text { Excluded as } \\
\text { clinically } \\
\text { unmotivated }\end{array}$ & $\begin{array}{c}\text { Excluded as } \\
-\mathbf{I . 5} \text { SD } \\
\text { outliers }\end{array}$ \\
\hline WAIS-R FSIQ & 0.87 & 0.82 & 0.86 \\
WMS-R General Memory & 1.03 & 1.03 & 1.03 \\
WMS-R Delay Recall & 0.68 & 0.68 & 0.75 \\
Index & & & \\
CVLT List A trials I-5 & 0.80 & 0.71 & 0.71 \\
CPT Distractibility correct & 0.40 & 0.70 & 0.84 \\
WCST categories & 0.55 & 0.62 & 0.55 \\
N-back 3 back correct & 0.54 & 0.52 & 0.54 \\
Category fluency & 1.33 & 1.33 & 1.28 \\
BLO & 0.54 & 0.55 & 0.51 \\
\hline
\end{tabular}

Table 4 Numbers and Percentages of Patients Improving by One or More SD Units to above Average on Different Tests of Cognitive Abilities

\begin{tabular}{|c|c|c|c|}
\hline & \multirow[b]{2}{*}{ I SD } & \multicolumn{2}{|c|}{ Percent normalized } \\
\hline & & $\begin{array}{l}\text { Stringent } \\
\text { method }\end{array}$ & $\begin{array}{l}\text { Lenient } \\
\text { method }\end{array}$ \\
\hline \multicolumn{4}{|l|}{ WAIS-R } \\
\hline FSIQ & 15 & 21.1 & 42.1 \\
\hline \multicolumn{4}{|l|}{ CPT } \\
\hline \multicolumn{4}{|l|}{ Distractibility } \\
\hline Correct & 4.5 & 29.4 & 29.4 \\
\hline Reaction time & 77.5 & 11.8 & 29.4 \\
\hline \multicolumn{4}{|l|}{ Trail Making Test } \\
\hline Form A & 12.5 & 11.8 & 17.6 \\
\hline Form B & 42.8 & 15.4 & 15.4 \\
\hline \multicolumn{4}{|l|}{ WMS-R } \\
\hline General memory index & 15 & 18.8 & 18.8 \\
\hline Delayed recall index & 15 & 23.5 & 23.5 \\
\hline \multicolumn{4}{|l|}{ CVLT } \\
\hline Long delay free recall & 2 & 16.7 & 16.7 \\
\hline Slope & 0.9 & 33.3 & 33.3 \\
\hline Recognition-discriminability & 11 & 33.3 & 33.3 \\
\hline BLO & 4 & 16.7 & 16.7 \\
\hline BFR & 3.96 & 18.8 & 18.8 \\
\hline \multicolumn{4}{|l|}{ WCST } \\
\hline Total number of categories & 1.6 & 16.7 & 16.7 \\
\hline Percent perseverative errors & 9.9 & 16.7 & 16.7 \\
\hline Letter fluency & 7 & 22.2 & 33.3 \\
\hline
\end{tabular}

all tests and $24.1 \%$ of the patients' scores could be considered to be normalized over all tests after applying the lenient method (see Table 4). The percent of patients normalized using the stringent method ranged between $11.8 \%$ (for WMS-R General Memory Index and CPT Distractibility RT) and $33.3 \%$ for CVLT (slope and discriminability). Using the lenient method $42.1 \%$ of the patients were normalized on the WAIS-R FSIQ.

\section{Symptoms}

Administration of atypical antipsychotics also produced a marked improvement in symptom measures relative to placebo. With respect to PANSS-positive symptoms, during atypical antipsychotic administration the patient's mean score of $13.0(\mathrm{SD}=3.9)$ was significantly less than their mean score of $17.4(\mathrm{SD}=5.1)$ measured during placebo withdrawal, $t(16)=3.14, p<0.006$. Likewise, with reference to PANSS-negative symptoms, during atypical antipsychotic administration the patients' mean score of $15.9(\mathrm{SD}=6.8)$ was significantly less than their mean score of 20.1 $(\mathrm{SD}=6.8) \quad$ measured during placebo withdrawal, $t(16)=2.36, p<0.031$. Additionally, with respect to the PANSS conceptual disorganization factor (Cuesta and Peralta, 1995; Dollfus et al, 1991), during atypical antipsychotic administration the patients' mean score of 4.3 $(\mathrm{SD}=2.3)$ was significantly less than their mean score of 6.0 $(\mathrm{SD}=2.5) \quad$ measured during placebo withdrawal, $t(16)=2.39, p<0.029$. The effect sizes obtained in the whole sample with respect to positive, negative, and disorganized symptoms were $0.76,0.57$, and 0.58 , respectively.

\section{Symptoms and Cognition}

We assessed the relation between symptom change (positive, negative, and disorganized) to change in those cognitive measures listed in Table 3 in a series of stepwise multiple regression analyses designed to maximize $r^{2}$. We also included IQ as a regressor as it too improved significantly and might have impacted on other cognitive functions. With the exception of the ability of the PANSS conceptual disorganization factor to predict significantly CPT number correct, $r^{2}=0.41, p=0.02$, neither individual symptom changes nor IQ, nor any combination significantly predicted change in cognitive measures $(p>0.10)$. Otherwise, $r^{2}$ was below 0.20 . Significant correlations of moderate strength obtained between symptom and cognitive change scores include: positive symptoms with measures of attention (CPT Distractibility number correct, $r=-0.59$ and commission errors, $r=0.51$ ), ocular-motor tracking (Trails A, $r=0.50$ ), delayed recall (WMS-R, $r=-0.53)$, and reaction time during 3-back $(r=0.65)$, negative symptoms with measures of attention (CPT Distractibility commission errors, $r=0.54$ ) and ocularmotor tracking (Trails A, $r=0.62$ ), and disorganization symptoms with measures of attention (CPT Distractibility correct, $r=-0.64$ ) and delayed recall (WMS-R, $r=-0.50$ ), with trends toward significance during reaction time for the 1-back $(r=0.55)$ and 3-back $(r=0.56)$. Correlations between symptom and cognitive change scores were in the $0.45>r>-0.41$ range and nonsignificant for all other measures. Detailed results of this set of analyses are available upon request.

\section{DISCUSSION}

Administration of atypical antipsychotic medication enhanced performance significantly across multiple cognitive domains assessed in patients with SC relative to their performance when receiving placebo during a period of antipsychotic withdrawal (3-4 weeks). The differences remained statistically significant even after a variety of exclusion criteria were applied to the data set in an attempt to eliminate poorly performing outliers that may have biased the results in favor of finding a benefit for 
antipsychotics. Although application of a Bonferroni correction procedure for multiple comparisons eliminated a number of the significant results, several striking enhancements in test performance due to atypical antipsychotic administration (WMS-R General Memory Index score and Category fluency scores) remained significant. The MANOVA main effect also argues in favor of an effect of medication upon various measures of different cognitive domains, albeit to an attenuated degree. The large percentage of statistically significant comparisons prior to application of the Bonferroni correction also suggests that atypical antipsychotic medication produces a breadth of cognitive enhancement in this sample. Clinical exclusion of 'unmotivated' performances failed to eliminate significant differences. Additionally, exclusion of outliers using a 1.5 SD cutoff also failed to eliminate statistically significant differences. Cognitive improvement with administration of atypical antipsychotics did not appear to be driven by those patients receiving clozapine therapy since most significant differences obtained for specific assessments still remained significant after exclusion of those patients receiving clozapine, thereby maintaining significant differences even with a reduction in sample size (data available upon request). Lack of differences between Time 1 and Time 2 performances argues in favor of medication effects over simple practice effects as being responsible for the cognitive improvement obtained in this study.

While administration of atypical antipsychotics also produced statistically significant reductions in symptoms as measured by PANSS ratings, the paucity of significant prediction of cognitive change by symptom change scores would suggest that the cognition did not improve due to direct attenuation of interfering symptom effects (but see discussion of unusual features and restoration of cognitive resources below). The series of regression analyses in our complete sample generally shows that the symptom change failed to predict cognitive change with the exception of the ability of the conceptual disorganization factor to predict CPT Distractibility correct. While this predictive factor may reflect some general improvement in gaining organized access to target representations in the face of distracters or competing stimuli, we were nevertheless struck by the overall lack of predictability. It is also important to note that none of the cognitive measures displaying moderately strong, significant correlations with symptom scores were variables showing the largest significant differences between administration of active and inactive atypical antipsychotics. The administration of adjunctive medication, such as valproic acid and benztropine, to a small number of patients in our sample during the active medication phase might have mild detrimental effects upon cognitive abilities and thus would operate to attenuate the findings. Use of a within-subject, double-blind, placebo-controlled, counterbalanced design also allowed partial examination of the hypothesis suggested by Carpenter and Gold (2003, 2002) that cognitive benefits following atypical antipsychotic administration are due to the absence of adverse cognitive effects associated with typical neuroleptics. By comparing atypical antipsychotic to placebo the effects of atypicals can be assessed relative to the disease state as opposed to a deficit state induced by typical neuroleptics. While patients in the present study displayed markedly better cognitive performance following administration of atypicals, a medication-free symptomatic state has its own inherent set of adverse factors that may produce cognitive deficits.

The results from this study support previous work that has demonstrated improvement in test scores following the administration of atypical antipsychotics. The sample of patients in the current study appeared to be representative of typical samples of patients with SC based on an approximate 10-point decline in IQ estimated by WRAT-R Reading and WAIS-R FSIQ difference scores. Also, patients were chronic and generally showed incomplete responses to treatment. Last, while some patients either withdrew from the study voluntarily or were withdrawn due to severe symptom exacerbation, the cognitive performances of these 12 patients, if it were not excluded for motivational reasons or as outliers, would most likely have been relatively poor due to frank disorganization and, therefore, would have resulted in improvements concomitant with antipsychotic administration.

Although many studies have shown improved performance on measures of attention following the administration of atypical antipsychotics (Hagger et al, 1993; McGurk et al, 1997; Serper and Chou, 1997; Stip and Lussier, 1996; Zahn et al, 1994), the present study obtained significant differences in measures of attention only following removal of outliers and prior to application of the Bonferroni correction. The present study is consistent with previous studies demonstrating improvement in some aspects of executive function following administration of atypical antipsychotics (Buchanan et al, 1994; Gallhofer et al, 1996; Green et al, 1997; Hagger et al, 1993; Lee et al, 1994; McGurk et al, 1997; Meyer-Lindenberg et al, 1997; Rossi et al, 1997) at least to the extent that significant differences were currently obtained prior to the application of the Bonferroni correction in the current study. However, many of the tests of executive function used across studies vary widely in their task demands. Indeed, while those tasks that demanded rapid updating of target selection ( $N$-back) and set shifting (WCST percent perseverative errors), both thought to be executive function subprocesses, did not improve significantly, WCST category formation, dependent on a 'win-stay' strategy (ie sensitive to positive feedback), improved markedly. Three previous studies have demonstrated improvements in episodic memory following administration of atypical antipsychotics (Kern et al, 1999; Lee et al, 1994; Potkin et al, 2001). In the present study, comprehensive and marked improvement in episodic memory was also observed (especially notable were learning slope improvements). Some studies have demonstrated improvement in simple working memory performance following atypical antipsychotic administration (Galletly et al, 1997; Green et al, 1997; Rossi et al, 1997); however, our study found no difference on WMS-R digit span forward between patients receiving antipsychotic medication and the same patients receiving placebo. Our study is also consistent with many studies that have shown improvement on measures of language production (letter and category fluency) following atypical antipsychotic administration (Buchanan et al, 1994; Galletly et al, 1997; Hagger et al, 1993; Hoff et al, 1996; Lee et al, 1994; Potkin et al, 2001). Also, our findings are consistent with two studies that show improvement in overall intellectual abilities (Fujii et al, 
1997; Potkin et al, 2001). Although we considered the possibility that changes in specific cognitive domains were simply a function of gaining in general intellectual efficiency, this could not be confirmed by a regression analysis. However, it is clear that the ability or inability to obtain statistically significant differences on any given measure may be due in part to the statistical properties of the measure and sensitivity to detect changes, and not necessarily direct improvement of underlying cognitive abilities.

Of all the 15 studies from the Keefe et al (1999) metanalysis, only three (Hagger et al, 1993; Serper and Chou, 1997; Zahn et al, 1994) incorporated a medicationfree period during which cognitive assessment was obtained. Although these studies did utilize a within-subject design, due to the administration of testing in a fixed order (placebo or medication-free prior to atypical antipsychotic) these studies may have been subject to order effects. Additionally, these studies used a medication-free phase that was relatively brief (7 days maximum), which would minimize the likelihood that prior antipsychotic effects had cleared. The current study utilized a within-subject design such that patients were able to serve as their own controls. This procedure not only provided additional power to detect significant differences for our moderate-sized sample, but it also allowed us to test for learning effects. The current study suggests that medication rather than simple learning effects account for observed cognitive improvement since cognitive improvement generally occurred following administration of atypical antipsychotic medication regardless of group assignment (atypical treatment first $v s$ placebo treatment first) and thus was consistent with the results of previous within-subject studies.

There are several unusual features in the results that bear discussion. First, scores changed on a test that is often thought to be stable, that is, IQ. Improvements were most marked in subtests of verbal abstraction and speed of processing (results available upon request), suggesting improvements at the level of conceptual organization and/ or attention. At the very least, the results imply that specific subprocesses account for gains, not reified notions of test labels. More generally, they may indicate that dopaminedependent signal-to-noise ratios may be more favorable in patients on antipsychotics, allowing for better performance. Second, beta weights for delineating the power of changes among symptom measures to predict changes in cognitive measures were consistently small and nonsignificant. While the results suggest that cognitive changes were not due to 'interference effects' caused by symptoms, they do not rule out the possibility that more general properties of neuronal signaling were improving, but were incompletely captured by both symptom and cognitive variables. Consistent with this is a third unusual feature of the data: improvements in cognition were relatively 'across the board' in the sense that they occurred over multiple domains of function. Again, the view that the effects of antipsychotic medication on neuromodualtory systems that bias networks toward more precise representations of stimuli and relations among representations, while speculative, cannot be dismissed out of hand. Of course, there are other less palatable possibilities. The results could be due to some unspecified confound or uncontrolled factor idiosyncratic to the present study or cohort, but we do not think this is highly probable because of the many prior reports of cognitive improvement with atypical antipsychotic treatment.

Clearly, global insults in brain function impair one's ability to perform in many domains of cognitive testing, and this manner of impairment can be reversed with clinical improvement in general brain function (Knights et al, 1991; Mandleberg and Brooks, 1975; Mayes et al, 1989; White et al, 1986). This raises the question of whether the improvements we observed represent a true cognitive enhancement per se or simply restoration of more effective utilization of cognitive resources that pre-existed the symptomatic state of psychosis and that were unavailable or dysfunctional during the more clinically disturbed state. In other words, while it has been difficult to demonstrate a clear correlation between clinical symptoms and cognitive deficits, the fact that symptomatic improvement occurs contemporaneously with improvement in varied and broad realms of cognition implies that these cognitive improvements are manifestations of improved brain function in a more general sense.

One limitation of the current study pertains to the variety of atypical antispsychotic medication administered. However, the aim of the study was not to assess the effect of any given atypical antipsychotic medication upon the cognitive abilities of patients with SC, but rather to determine whether atypical antipsychotic medications as a general class produced cognitive improvement relative to an antipsychotic-free period (be it based on appropriate dosing, receptor pharmacology, or other unknown factors).

The extent of improvement across cognitive domains was not unexpected based on our current understanding of antipsychotic action and prior studies of atypical antipsychotics. What was unexpected was the magnitude of the effect based on effect size units. The implications of these results invite skepticism. Nevertheless, given the hardiness of the results to various manipulations designed to reduce the impact of confounds, the findings consistently support the idea that atypical antipsychotic medication used in doses that are low compared to historical doses of typical neuroleptics and that do not produce extrapyramidal side effects frequently have direct effects on tests of cognition at least insofar as improvement in cognitive test scores was not secondary to outlier effects or symptom and motivation changes.

\section{ACKNOWLEDGEMENTS}

This paper was presented in part at the 39th Annual meeting of the American College of Neuropsychopharmacology, December 2000.

\section{REFERENCES}

Benton AL, Hamsher KD, Varney NR, Spreen O (1983). Contributions to Neuropsychological Assessment. Oxford: New York.

Bilder RM, Goldman RS, Volavka J, Czobor P, Hoptman M, Sheitman B et al (2002). Neurocognitive effects of clozapine, olanzapine, risperidone, and haloperidol in patients with chronic schizophrenia or schizoaffective disorder. Am J Psychiatry 159: 1018-1028.

Buchanan RW, Holstein C, Breier A (1994). The comparative efficacy and long-term effect of clozapine treatment on 
neuropsychological test performance. Biol Psychiatry 36: 717-725.

Callicott JH, Mattay VS, Bertolino A, Finn K, Coppola R, Frank JA et al (1999). Physiological characteristics of capacity constraints in working memory as revealed by functional MRI. Cereb Cortex 9: 20-26.

Carpenter WT, Gold JM (2002). Another view of therapy for cognition in schizophrenia. Biol Psychiatry 51: 969-971.

Carpenter WT, Gold JM (2003). Reply to: atypical antipsychotic drugs improve cognition in schizophrenia. Biol Psychiatry 53: 267-268.

Cole JO, Klerman GL, Goldberg SC, Clyde DJ, Davidson EM, Kayce MM et al (1964). Phenothiazine treatment in acute schizophrenia. Arch Gen Psychiatry 10: 246-261.

Cuesta MJ, Peralta V (1995). Psychopathological dimensions in schizophrenia. Schizophr Bull 21: 473-482.

Davis JM, Chen N (2001). The effects of olanzapine on the 5 dimensions of schizophrenia derived by factor analysis: combined results of the North American and international trials. J Clin Psychiatry 62: 757-771.

Davis JM, Chen N (2002). Clinical profile of an atypical antipsychotic: risperidone. Schizophr Bull 28: 43-61.

Delis DC, Kramer JH, Kaplan E, Ober BA (1987). California Verbal Learning Test Manual. Psychological Corp.: New York.

Dollfus S, Petit M, Lesieur P, Menard J (1991). Principalcomponent analysis of PANSS and SANS-SAPS global ratings in schizophrenic patients. Eur Psychiatry 6: 251-259.

Farde L, Wiesel FA, Nordstrom AL, Sedvall G (1989). D1- and D2dopamine receptor occupancy during treatment with conventional and atypical neuroleptics. Psychopharmacology (Berl) 99(Suppl): S28-S31.

Fujii DE, Ahmed I, Jokumsen M, Compton JM (1997). The effects of clozapine on cognitive functioning in treatment-resistant schizophrenic patients. J Neuropsychiatry Clin Neurosci 9: 240-245.

Galletly CA, Clark CR, McFarlane AC, Weber DL (1997). Relationships between changes in symptom ratings, neurophysiological test performance and quality of life in schizophrenic patients treated with clozapine. Psychiatry Res 72: 161-166.

Gallhofer B, Bauer U, Lis S, Krieger S, Gruppe H (1996). Cognitive dysfunction in schizophrenia: comparison of treatment with atypical antipsychotic agents and conventional neuroleptic drugs. Eur Neuropsychopharmacol 6(Suppl 2): S13-S20.

Geddes J, Freemantle N, Harrison P, Bebbington P (2000). Atypical antipsychotics in the treatment of schizophrenia: systematic overview and meta-regression analysis. BMJ 321: 1371-1376.

Goldberg TE, Egan MF, Gscheidle T, Coppola R, Weickert T, Kolachana BS et al (in press). Executive subprocesses in Working Memory: Relationships with COMT Val108/158 Met genotype and genetic risk for Schizophrenia. Arch Gen Psychiatry.

Goldberg TE, Weinberger DR (1996). Effects of neuroleptic medications on the cognition of patients with schizophrenia: a review of recent studies. J Clin Psychiatry 57(Suppl 9): 62-65.

Gordon M (1987). Instruction Manual for the Gordon Diagnostic System. Gordon Systems: DeWitt, NY.

Gordon M, McClure FD, Aylward GP (1996). The Gordon Diagnostic System Interpretive Guide, 3rd edn GSI Publications: DeWitt, NY.

Green MF, Marshall Jr BD, Wirshing WC, Ames D, Marder SR, McGurk S et al (1997). Does risperidone improve verbal working memory in treatment-resistant schizophrenia? Am J Psychiatry 154: 799-804.

Hagger C, Buckley P, Kenny JT, Friedman L, Ubogy D, Meltzer HY (1993). Improvement in cognitive functions and psychiatric symptoms in treatment-refractory schizophrenic patients receiving clozapine. Biol Psychiatry 34: 702-712.
Heaton RK, Chelune GJ, Talley JL, Kay GG, Curtiss G (1993). Wisconsin Card Sorting Test Manual: Revised and Expanded. Psychological Assessment Resources: Odessa, FL.

Hoff AL, Faustman WO, Wieneke M, Espinoza S, Costa M, Wolkowitz O et al (1996). The effects of clozapine on symptom reduction, neurocognitive function, and clinical management in treatment-refractory state hospital schizophrenic inpatients. Neuropsychopharmacology 15: 361-369.

Jastak S, Wilkinson GS (1984). The Wide Range Achievement TestRevised Administration Manual. Jastak Associates: Wilmington. Kaufman AS (1990). Assessing Adolescent and Adult Intelligence. Allyn and Bacon: Needham, MA.

Kay SR, Fiszbein A, Opler LA (1987). The positive and negative syndrome scale (PANSS) for schizophrenia. Schizophr Bull 13: 261-276.

Keefe RS, Silva SG, Perkins DO, Lieberman JA (1999). The effects of atypical antipsychotic drugs on neurocognitive impairment in schizophrenia: a review and meta-analysis. Schizophr Bull 25: 201-222.

Kern RS, Green MF, Marshall Jr BD, Wirshing WC, Wirshing D, McGurk SR et al (1999). Risperidone versus haloperidol on secondary memory: can newer medications aid learning? Schizophr Bull 25: 223-232.

Knights RM, Ivan LP, Ventureyra EC, Bentivoglio C, Stoddart C, Winogron W et al (1991). The effects of head injury in children on neuropsychological and behavioural functioning. Brain Injury 5: 339-351.

Lee MA, Thompson PA, Meltzer HY (1994). Effects of clozapine on cognitive function in schizophrenia. J Clin Psychiatry 55(Suppl B): 82-87.

Mandleberg IA, Brooks DN (1975). Cognitive recovery after severe head injury. 1. Serial testing on the Wechsler Adult Intelligence Scale. J Neurol Neurosurg Psychiatry 38: 1121-1126.

Mayes SD, Pelco LE, Campbell CJ (1989). Relationships among pre- and post-injury intelligence, length of coma and age in individuals with severe closed-head injuries. Brain Injury 3: 301-313.

McGurk S, Green M, Wirshing W, Ames D, Marshall BJ, Marder A et al (1997). The effects of risperidone vs haloperidol on cognitive functioning in treatment resistant schizophrenia: The Trail Making Test. CNS Spectrums 2: 60-64.

Meltzer HY (1989). Clinical studies on the mechanism of action of clozapine: the dopamine-serotonin hypothesis of schizophrenia. Psychopharmacology (Berl) 99(Suppl): S18-S27.

Meltzer HY (1992). Dimensions of outcome with clozapine. $\mathrm{Br} J$ Psychiatry 160(Suppl 17): 46-53.

Meltzer HY, Bastani B, Ramirez L, Matsubara S (1989a). Clozapine: new research on efficacy and mechanism of action. Eur Arch Psychiatry Neurol Sci 238: 332-339.

Meltzer HY, Matsubara S, Lee JC (1989b). Classification of typical and atypical antipsychotic drugs on the basis of dopamine D-1, D-2 and serotonin2 pKi values. J Pharmacol Exp Ther 251: 238246.

Meltzer HY, Matsubara S, Lee JC (1989c). The ratios of serotonin2 and dopamine2 affinities differentiate atypical and typical antipsychotic drugs. Psychopharmacol Bull 25: 390-392.

Meltzer HY, McGurk SR (1999). The effects of clozapine, risperidone, and olanzapine on cognitive function in schizophrenia. Schizophr Bull 25: 233-255.

Meyer-Lindenberg A, Gruppe H, Bauer U, Lis S, Krieger S, Gallhofer B (1997). Improvement of cognitive function in schizophrenic patients receiving clozapine or zotepine: results from a double-blind study. Pharmacopsychiatry 30: 35-42.

Missar CD, Gold JM, Goldberg TE (1994). WAIS-R short forms in chronic schizophrenia. Schizophr Res 12: 247-250.

Palmer B, Heaton R, Paulsen J, Kuck J, Braff D, Harris M et al (1997). Is it possible to be schizophrenic yet neuropsychologically normal? Neuropsychology 11: 437-446. 
Potkin SG, Fleming K, Jin Y, Gulasekaram B (2001). Clozapine enhances neurocognition and clinical symptomatology more than standard neuroleptics. J Clin Psychopharmacol 21: 479-483.

Purdon SE, Labelle A, Boulay L (2001). Neuropsychological change in schizophrenia after 6 weeks of clozapine. Schizophr Res 48: 57-67.

Reitan RM (1986). Trail-Making Test Manual for Administration and Scoring. Reitan Neuropsychology Laboratory: Tucson, AZ.

Richelson E (1984). Neuroleptic affinities for human brain receptors and their use in predicting adverse effects. J Clin Psychiatry 45: 331-336.

Rossi A, Mancini F, Stratta P, Mattei P, Gismondi R, Pozzi F et al (1997). Risperidone, negative symptoms and cognitive deficit in schizophrenia: an open study. Acta Psychiatr Scand 95: 40-43.

Seeman P (1987). Dopamine receptors and the dopamine hypothesis of schizophrenia. Synapse 1: 133-152.

Serper M, Chou J (1997). Novel improve attentional functioning in schizophrenic patients: ziprasidone and aripiprazole. CNS Spectrums 2: 56-59.

Spreen O, Strauss E (1991). A Compendium of Neuropsychological Tests. Oxford: New York.

Stip E, Lussier I (1996). The effect of risperidone on cognition in patients with schizophrenia. Can J Psychiatry 41(8 Suppl 2): S35-S40.

Wechsler D (1981). Wechsler Adult Intelligence Scale-Revised Manual. The Psychological Corp.: San Antonio.

Wechsler D (1987). Wechsler Memory Scale-Revised Manual. Psychological Corp: New York.

Weickert TW, Goldberg TE, Gold JM, Bigelow LB, Egan MF, Weinberger DR (2000). Cognitive impairments in patients with schizophrenia displaying preserved and compromised intellect. Arch Gen Psychiatry 57: 907-913.

White PD, Lishman WA, Wyke MA (1986). Phaeochromocytoma as a cause of reversible dementia. J Neurol Neurosurg Psychiatry 49: 1449-1451.

Zahn TP, Pickar D, Haier RJ (1994). Effects of clozapine, fluphenazine, and placebo on reaction time measures of attention and sensory dominance in schizophrenia. Schizophr Res 13: 133-144. 